\title{
Estudo anatômico do túnel radial e suas implicações nas síndromes compressivas*
}

\section{Anatomical Study of Radial Tunnel and its Clinical Implications in Compressive Syndromes}

\author{
Edie Benedito Caetano ${ }^{10}$ Luiz Angelo Vieira ${ }^{1}$ João José Sabongi Neto ${ }^{2}$ \\ Maurício Benedito Ferreira Caetano ${ }^{2}$ Rodrigo Guerra Sabongi ${ }^{3}$ Yuri da Cunha Nakamichi ${ }^{1}$
}

${ }^{1}$ Faculdade de Ciências Médicas e da Saúde, Pontifícia Universidade

Endereço para correspondência Edie Benedito Caetano, PhD, Católica de São Paulo, Sorocaba, SP, Brasil

Faculdade de Ciências Médicas e da Saúde, Pontifícia Universidade Católica de São Paulo, Rua Joubert Wey, 290, Sorocaba, SP 18030-070,

2 Serviço de Cirurgia da Mão, Conjunto Hospitalar de Sorocaba,

Sorocaba, SP, Brasil

3 Universidade Federal do Estado de São Paulo (UNIFESP), São Paulo, Brasil (e-mail: ediecaetano@uol.com.br).

SP, Brasil

Rev Bras Ortop 2020;55(1):27-32.

\section{Resumo \\ Palavras-chave \\ - nervo radial \\ - neuropatia radial \\ - síndromes de compressão nervosa \\ - cadáver \\ Objetivo O objetivo do presente estudo foi analisar as estruturas contidas no túnel radial que podem causar neuropatia compressiva do nervo interósseo posterior. \\ Métodos Foram dissecados 30 membros de 15 cadáveres adultos, preparados por injeção intra-arterial de uma solução de glicerina e formol a $10 \%$. Todos do sexo masculino, pertencentes ao laboratório de anatomia desta instituição. \\ Resultados O ramo para o músculo supinador originou-se do nervo interósseo posterior em todos os membros. Identificamos a arcada de Frohse com uma constituição fibrosa bem desenvolvida em 22 dos 30 membros dissecados (73\%) e de constituição muscular em 8 (23\%) A margem distal do músculo supinador apresentou consistência fibrosa em 7 dos 30 membros (23,5\%) e uma aparência muscular em 23 (76,5\%). Na margem proximal do músculo extensor radial curto do carpo, identificamos a arcada fibrosa em 18 membros (60\%); em 9 (30\%), notamos a arcada de constituição muscular; e em três (10\%) havia apenas a inserção radial, de maneira que não formava a arcada. \\ Conclusão A arcada de Frohse e a arcada formada pelas origens do músculo extensor radial curto do carpo são estruturas anatômicas normais em cadáveres adultos. No entanto, sob o ponto de vista clínico, essas estruturas têm potencial para causar a compressão do nervo interósseo posterior.}

Abstract

Objective The purpose of the present study was to analyze the structures in the radial tunnel that can cause posterior interosseous nerve entrapment.

Methods A total of 30 members of 15 adult cadavers prepared by intra-arterial injection of a $10 \%$ solution of glycerol and formalin were dissected. All were male, belonging to the laboratory of anatomy of this institution.

Trabalho realizado na Pontifícia Universidade Católica de São Paulo,

Faculdade de Ciências Médicas e da Saúde, Sorocaba, SP, Brasil.

recebido

27 de Agosto de 2018

aceito

08 de Janeiro de 2019
DOI https://doi.org/

10.1055/s-0039-1700821. ISSN 0102-3616.
Copyright $\odot 2020$ by Sociedade Brasileira License terms de Ortopedia e Traumatologia. Published by Thieme Revinter Publicações Ltda, Rio de Janeiro, Brazil 


\author{
Keywords \\ - radial nerve \\ - radial neuropathy \\ - nerve compression \\ syndromes \\ - cadaver
}

Results The branch for the supinator muscle originated from the posterior interosseous nerve in all limbs. We identified the Frohse arcade with a well-developed fibrous constitution in 22 of the 30 dissected limbs (73\%) and of muscular constitution in 8 (27\%). The distal margin of the supinator muscle presented fibrous consistency in 7 of the 30 limbs (23.5\%) and muscular appearance in 23 (76.5\%). In the proximal margin of the extensor carpi radialis brevis muscle, we identified the fibrous arch in 18 limbs $(60 \%)$; in $9(30 \%)$ we noticed the arcade of muscular constitution; in $3(10 \%)$ there was only the radial insertion, so that it did not form the arcade.

Conclusion The Frohse arcade and the arcade formed by the origins of the extensor carpi radialis brevis are normal anatomical structures in adult cadavers. However, from the clinical point of view, these structures have the potential to cause entrapment of the posterior interosseous nerve.

\section{Introdução}

O nervo radial (NR) é o principal dentre os que se originam do fascículo posterior do plexo braquial. Ele inerva todos os músculos do compartimento posterior do braço e do antebraço. Ele passa do compartimento posterior para o anterior contornando o sulco do NR no úmero, e passa pelo septo intermuscular entre os músculos braquial (B) medialmente e braquiorradial (BR) lateralmente. Segue distalmente, emergindo entre os músculos BR e extensor radial longo do carpo (ERLC). Divide-se em ramo superficial do NR e nervo interósseo posterior (NIP), também chamado ramo profundo do NR.

O túnel radial é uma estrutura músculo-aponeurótica que se estende desde o epicôndilo lateral do úmero até a margem distal do músculo supinador $(S) .{ }^{1,2}$ A compressão do NIP no túnel radial pode resultar em duas apresentações clínicas distintas: a síndrome do NIP, que resulta em paralisia motora, enquanto a síndrome do túnel radial resulta em sintomas sensitivos, dor na face lateral do cotovelo e antebraço, ocasionalmente diagnosticada erroneamente como epicondilite lateral do cotovelo. ${ }^{1}$

Pode parecer paradoxal que a compressão de um nervo motor possa causar dor como o sintoma inicial. No entanto, além das fibras motoras, o NIP é constituído por fibras aferentes sensoriais destinadas à articulação do punho e por fibras aferentes destinadas aos músculos que elas suprem. ${ }^{3}$ Sensações de dor ou "peso" podem muito bem ser mediadas por esses fatores. ${ }^{3} \mathrm{O} \mathrm{S}$ é composto por duas cabeças, superficial e profunda; o NIP posiciona-se entre elas. A borda proximal da cabeça superficial do $S$ pode formar uma arcada fibrosa de espessura e comprimento variável também conhecida como arcada de Frohse (AF), descrita em 1908 por Frohse et al, ${ }^{4}$ e tem sido descrita como o local mais comum de compressão do NIP. ${ }^{2,5,6}$ Esta compressão nervosa pode ser agravada com movimentos repetitivos de pronação e supinação do antebraço. Outras estruturas, como a margem proximal fibrosa do músculo extensor radial curto do carpo (ERCC), que tem duas origens, uma óssea no lado radial e outra fascial no lado ulnar, formando uma arcada, por onde cursa o NIP, a qual tem sido envolvida no desenvolvimento da síndrome compressiva do NIP, posicionando-se proximalmente ou às vezes distalmente em relação à $\mathrm{AF}^{1}{ }^{1} \mathrm{O}$ objeto do presente estudo foi identificar quais as estruturas contidas no túnel radial que potencialmente podem ser responsáveis pelo desenvolvimento da neuropatia compressiva do NIP.

\section{Material e Métodos}

O presente estudo baseou-se na dissecção de 30 membros de 15 cadáveres, todos do sexo masculino. Foram preparados por injeção intra-arterial de uma solução de glicerina e formol a $10 \%$. Dissecamos quatro membros de cadáveres de dois fetos a termo com a finalidade de avaliar a composição do $S$, ou seja, se as arcadas fetais seriam apenas musculares ou apresentariam alguma composição fibrosa. Estes quatro membros não foram incluídos no presente estudo. Cada antebraço foi dissecado com o cotovelo em extensão, o punho em posição neutra e antebraço em pronação. Nenhum dos cadáveres apresentou evidências de deformidades, procedimentos cirúrgicos anteriores ou lesões traumáticas na área estudada. Removemos a pele e a fáscia da superfície anterolateral do terço distal do braço, do antebraço e do punho.

O NR foi identificado no braço entre os músculos B e BR, e dissecado de proximal para distal. Os tendões dos músculos BR, ERLC e ERCC foram secionados em seu terço distal, separados das conexões fibrosas que os unia para facilitar a identificação dos ramos nervosos. Os ramos destinados aos músculos BR, ERLC, ERCC, NIP e ramos para o $\mathrm{S}$ foram dissecados. As estruturas vasculares não foram preservadas para facilitar a dissecção dos nervos. Utilizamos em certas fases da dissecção uma lupa de 2,5 x de aumento. A relação do NIP com o túnel fibroso formado pela margem proximal do músculo ERCC, com a AF e com a margem distal do $\mathrm{S}$ foram estudadas e classificadas como fibrosas se tivessem textura fibrotendinosa; se a aparência fosse muscular ou translúcida, foram definidas como musculares. O presente trabalho foi aprovado pela Comissão de Ética com o parecer número 2.207.258.

\section{Resultados}

Para facilitar o entendimento do que é proximal, distal, medial e lateral, colocamos o indicativo nas figuras. Identificamos a AF com uma constituição fibrosa bem desenvolvida 

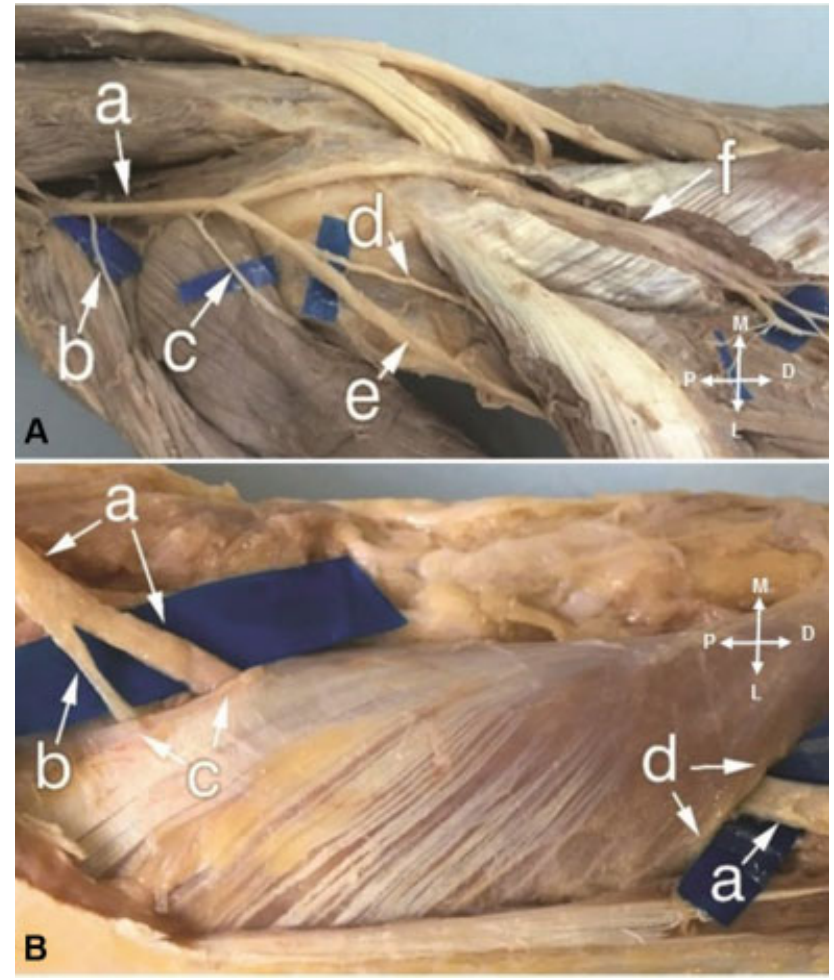

Fig. 1 (A) - (a) nervo mediano (NM); (b) ramo para BR; (c) ramo para ERLC; (d); ramo para ERCC; (e) ramo superficial do NR; (f) NIP. (B) - (a) NIP; (b) Ramo para o S; (c) AF fibrosa; (d) margem distal muscular do S.

em 22 membros (73\%) (-Fig. 1A e 1B) e de constituição muscular em 8 (27\%) (-Fig. 2A e 2B). A margem distal do $S$ apresentou-se de consistência fibrosa em 7 dos 30 membros (23,5\%), (-Fig. 3A) e de aparência muscular em 23
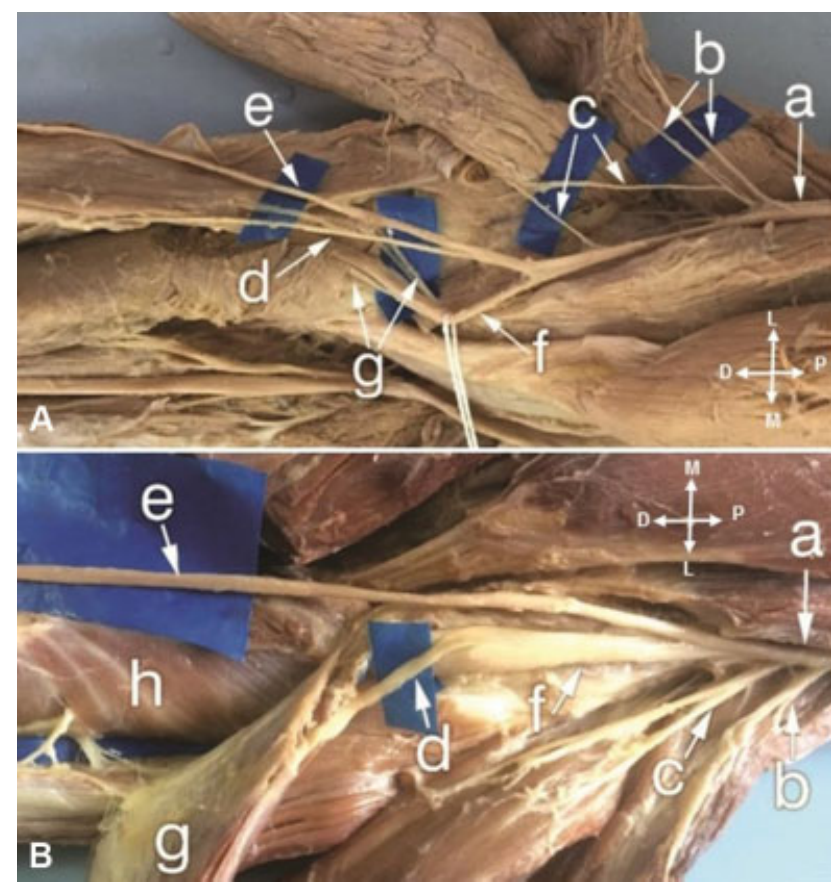

Fig. 2 (A) - (a) NM; (b) ramos para BR; (c) ramos para ERLC; (d); ramo para ERCC; (e) RSNR; (f) NIP; (g) ramos para o S. (B) - (a) NM; (b) ramo para BR; (c) ramo para ERLC; (d); ramo para ERCC; (e) NIP; (f) ERCC; (g) S.

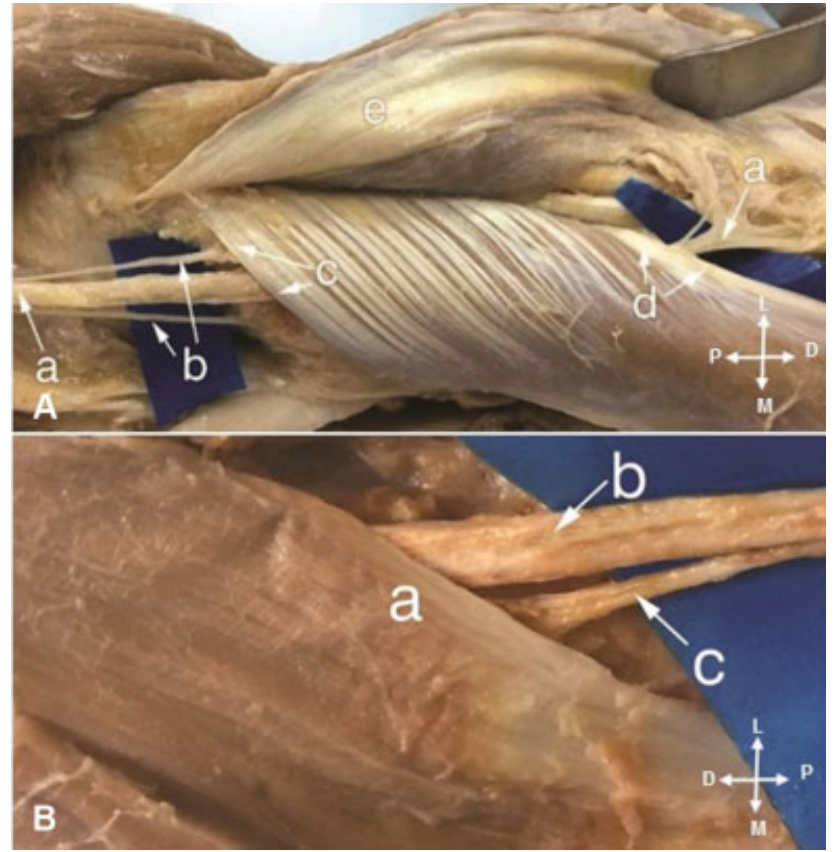

Fig. 3 (A) - (a) NIP; (b) Ramos para o S proximal à AF; (c) AF fibrosa; (d) margem distal fibrosa do S. (B)- (a) margem fibrosa do ERCC; (b) NIP; (c) ramo para o $\mathrm{S}$.

membros (76,5\%) (-Fig. 1B). Na margem proximal do músculo ERCC, identificamos uma arcada constituída através uma origem óssea do lado radial e fascial do lado ulnar; entre as duas origens havia um túnel por onde cursava o NIP. Em 18 membros (60\%) identificamos a arcada fibrosa ( - Fig. 3B); em 9 (30\%), notamos a arcada de constituição muscular (-Fig. 4A); e em 3 (10\%) membros havia apenas a inserção radial, de maneira que não formava a arcada (-Fig. 4B). A arcada do músculo ERCC posicionou-se proximalmente à $\mathrm{AF}$ em 17 membros (57\%), em contato direto com o NIP, apoiando-se sobre ele (-Fig. 3B e 4A), mas em apenas 6 (20\%) consideramos uma arcada com espessura e estrutura macroscópica visível, com consistência convincente para o NIP. Em 4 (13,5\%) membros, posicionou-se no mesmo nível da AF (-Fig. 5A), e em 6 membros (20\%) identificamos a arcada posicionar-se distalmente em relação à $\mathrm{AF}$ de forma que se apoiava sobre o $\mathrm{S}$ e não sobre o NIP (-Fig. 5B).

\section{Discussão}

Kopell et $\mathrm{al}^{7}$ descreveram pela primeira vez a compressão do NIP no túnel radial. Spinner ${ }^{2}$ relata que estudou 25 membros de cadáveres adultos e 10 membros de fetos a termo. A ausência da AF fibrosa nos fetos, em contraste com a incidência de $30 \%$ de arcada fibrosa em cadáveres de adultos, sugerem que o componente fibroso da arcada se forma na parte superficial do $\mathrm{S}$ em resposta ao movimento repetitivo de pronação e supinação do antebraço. ${ }^{2}$ Tivemos a curiosidade e dissecamos quatro antebraços de fetos e realmente nenhum vestígio de tecido fibroso no $\mathrm{S}$ foi identificado. Werner ${ }^{8}$ relatou que observou 39 (65\%) arcadas fibrosas em 60 cadáveres não selecionados, e em 80 (89\%) de 90 pacientes tratados cirurgicamente. Lister et $\mathrm{al}^{9}$ relatam uma série de 20 casos operados, 

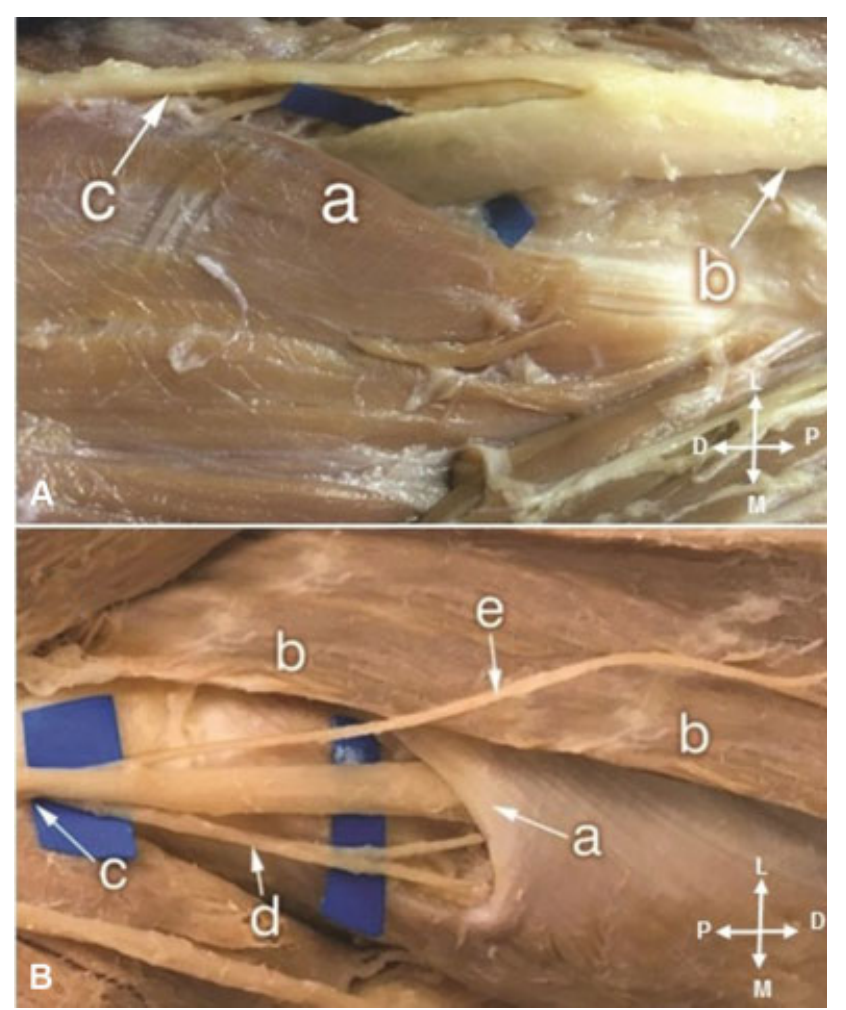

Fig. 4 (A) - (a) margem muscular do ERCC; (b) NIP; (c) ramo para o S. (B) - (a) AF fibrosa; ERCC (apenas com inserção óssea sem formação da arcada); (c) NIP; (d) ramo para o S; (e) RSNR.

todos unilaterais, e em todos identificaram a AF espessa de constituição fibrosa. Papadopoulos et $\mathrm{al}^{10}$ analisaram uma série de 120 membros de cadáveres e observaram que a $\mathrm{AF}$ se apresentou fibrosa em 61 (51\%).

Prasartritha et al ${ }^{11}$ dissecaram 60 membros de 30 cadáveres, identificaram a AF de constituição membranosa em 26 (43\%) e fibrotendinosa em 34 (57\%). Riffaud et al. ${ }^{12}$ dissecaram 25 membros de cadáveres, em 23 (95\%) identificaram a AF de constituição fibrosa. Ozkan et $\mathrm{al}^{13}$ realizaram um estudo anatômico do túnel radial em 60 membros de cadáveres e identificaram a AF fibrosa em 48 (80\%). Ebraheim et al ${ }^{14}$ dissecaram 20 membros de cadáveres e identificaram a arcada fibrosa em $14(70 \%)$. Konjengbam et $\mathrm{al}^{15}$ registraram a AF fibrosa em 40 (87\%) dos 46 membros de cadáveres. Ozturk et al ${ }^{6}$ identificaram a AF tendinosa em 48 (87\%) dos membros de cadáveres adultos que estudaram. Meng et al ${ }^{16}$ examinaram 21 membros de cadáveres e identificaram a arcada tendinosa em 16 (71\%) e membranosa em 5 (29\%). Rinker et $\mathrm{al}^{3}$ informam que operaram 79 pacientes, e em todos identificaram uma AF fibrosa. Clavert et al. ${ }^{5}$ informam que a AF tinha forma semicircular e se mostrou de consistência tendinosa em 26 membros (87\%) e de consistência membranosa nos 4 restantes (13\%). Em nosso estudo, a AF de conformação fibrosa foi identificada em 22 membros (73\%) e muscular em 8 (27\%), e não houve diferença estatistica entre os antímeros direito e esquerdo. As explicações pela discrepância dos números só podem ser explicadas pela diferente forma de interpretação da constituição da AF.

A margem distal do $\mathrm{S}$ representa o final do túnel radial, e excepcionalmente, pode ser a causa da compressão do nervo interósseo posterior. A margem distal do $\mathrm{S}$ de consistência
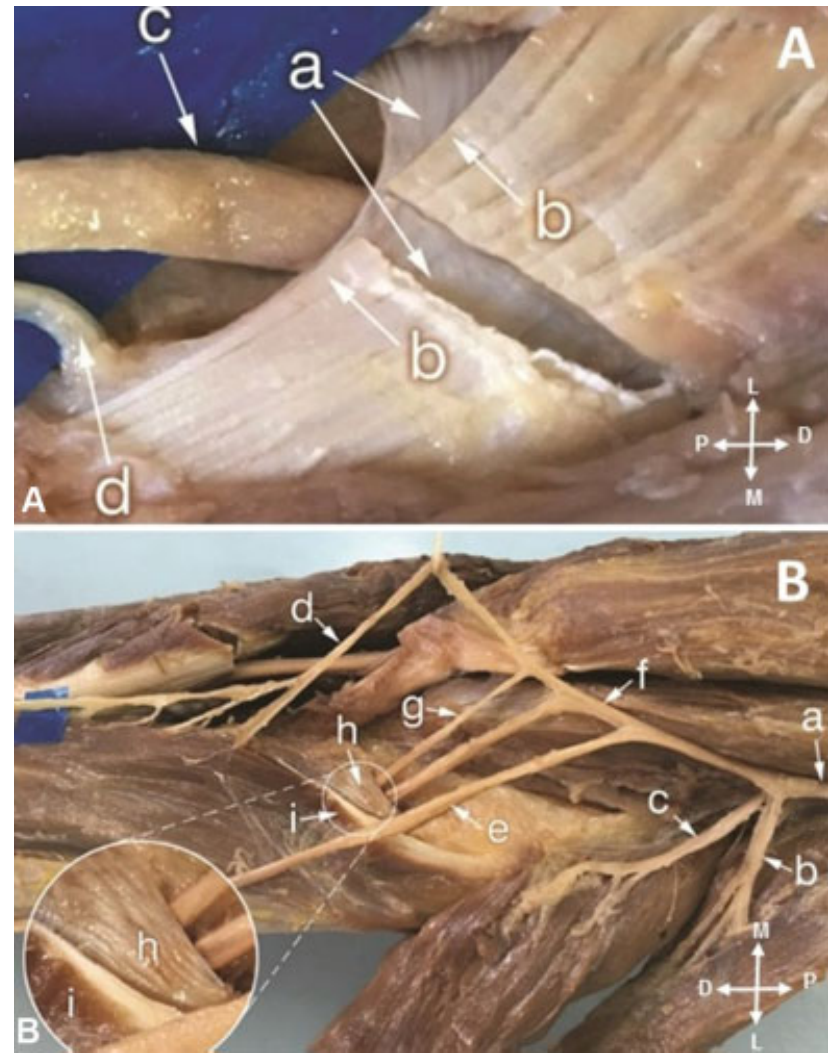

Fig. 5 (A) - (a) AF fibrosa e (b) arcada do ERCC (seccionada) no mesmo nível; (c) NIP; (d) ramo para o S. (B) - a) NM; (b) ramo para BR; (c) ramo para ERLC; (d); ramo para ERCC; (e) RSNR; (f) NIP; (g) S. (h) AF, situada proximalmente à arcada do ERCC (i).

fibrosa foi identificada por Konjengbam et $\mathrm{al}^{15}$ em 30 (65\%) de 46 membros dissecados, e por Riffaud et al ${ }^{12}$ em apenas 3 (7,5\%) de 25 membros. Identificamos a margem distal do $\mathrm{S}$ com consistência fibrosa em 7 dos 30 membros (23,5\%). A explicação pela discrepância dos números é a mesma em relação à $\mathrm{AF}$, somente podem ser explicadas pelas diferentes formas de interpretação. Além da AF, a compressão nervosa do NIP pode ser causada por diversas estruturas. A segunda em ordem de frequência é a arcada músculotendínea constituída pelas duas origens do músculo ERCC, uma origem óssea no lado radial e uma origem fascial no lado ulnar, entre as quais cursa o NIP. Essa arcada pode se situar proximalmente ou distalmente à AF. Riffaud et a ${ }^{12}$ relatam que estudaram 25 membros de cadáveres e identificaram a arcada do músculo ERCC em todos, posicionando-se sempre proximalmente à $\mathrm{AF}$. Papadopoulos et $\mathrm{al}^{10}$ analisaram uma série de 120 membros de cadáveres e observaram a arcada do músculo ERCC de constituição fibrosa em 90\% dos membros. Konjengbam et $\mathrm{al}^{15}$ identificaram a margem súpero-medial do ERCC tendinosa em 36 de 46 (78\%) membros de cadáveres. Clavert et $\mathrm{al}^{5}$ examinaram 30 membros de cadáveres preparados previamente, e em 4 membros (13\%), identificaram a presença de um componente fibroso do músculo ERCC envolvendo o NIP; eles informam, no entanto, que não registraram evidência macroscópica da compressão do NIP pela AF e nem por outras estruturas adjacentes. Nayak et a $1{ }^{17}$ relatam que dissecaram 72 membros de cadáveres e identificaram uma arcada tendinosa do ERCC em 21 membros (29,1\%) e muscular 
Estudo Anatômico do Túnel Radial Caetano et al. 31

Tabela 1 Sumário da literatura envolvendo a incidência da arcada de Frohse e margem distal do supinador de acordo com os autores

\begin{tabular}{|c|c|c|c|c|c|c|c|}
\hline Autores & $\begin{array}{l}\text { AF } \\
\text { fibrosa }\end{array}$ & $\begin{array}{l}\text { AF } \\
\text { muscular }\end{array}$ & $\begin{array}{l}\text { MD } \\
\text { fibrosa }\end{array}$ & $\begin{array}{l}\text { MD } \\
\text { muscular }\end{array}$ & $\begin{array}{l}\text { Tipo de } \\
\text { estudo }\end{array}$ & $\begin{array}{l}\text { Número } \\
\text { de casos }\end{array}$ & Ano \\
\hline Spinner $^{2}$ & $07(30 \%)$ & $18(70 \%)$ & - & - & anatômico & 25 & 1968 \\
\hline Werner $^{8}$ & 80 (89\%) & $10(11 \%)$ & - & - & cirúrgico & 90 & 1979 \\
\hline Lister et $\mathrm{al}^{9}$ & $20(100 \%)$ & $0(0 \%)$ & - & - & cirúrgico & 20 & 1979 \\
\hline Papadopoulos et al ${ }^{10}$ & $61(51 \%)$ & $59(49 \%)$ & - & - & anatômico & 120 & 1989 \\
\hline Prasartritha et al ${ }^{11}$ & $34(57 \%)$ & $26(43 \%)$ & - & - & anatômico & 60 & 1993 \\
\hline Riffaud et al ${ }^{12}$ & $23(95 \%)$ & $2(5 \%)$ & $3(7,5 \%)$ & $22(92,5 \%)$ & anatômico & 25 & 1999 \\
\hline Ozkan et al ${ }^{13}$ & $48(80 \%)$ & $12(20 \%)$ & - & - & anatômico & 60 & 1999 \\
\hline Ebraheim et al $^{14}$ & $14(70 \%)$ & $6(30 \%)$ & - & - & anatômico & 20 & 2000 \\
\hline Konjengbam et al ${ }^{15}$ & $40(87 \%)$ & $6(13 \%)$ & $30(65 \%)$ & $16(35 \%)$ & anatômico & 46 & 2004 \\
\hline Rinker et $\mathrm{al}^{3}$ & 79 (100\%) & $0(0 \%)$ & - & - & cirúrgico & 79 & 2004 \\
\hline Ozturk et $\mathrm{al}^{6}$ & $48(87 \%)$ & $07(13 \%)$ & - & - & anatômico & 55 & 2005 \\
\hline Clavert et $\mathrm{al}^{5}$ & $26(87 \%)$ & 34 (57\%) & - & - & anatômico & 30 & 2009 \\
\hline Meng et al ${ }^{16}$ & $16(71 \%)$ & 05 (29\%) & & & anatômico & 21 & 2015 \\
\hline Caetano et al $^{*}$ & 22 (73\%) & $08(17 \%)$ & $7(23,5 \%)$ & $23(76,5 \%)$ & anatômico & 30 & 2017 \\
\hline
\end{tabular}

Abreviações: AF, arcada de Frohse; MD, margem distal do supinador.

*Dados deste manuscrito.

em 8 (11,1\%), e não identificaram a presença da arcada em 43 membros (59,7\%); eles informam que a arcada do ERCC envolve o NIP, podendo causar sua compressão. Vergara-Amador et al ${ }^{1}$ relatam que estudaram 21 membros de cadáveres e identificaram uma arcada fibrotendinosa na margem súpero-medial do músculo ERCC em 20 membros (95,2\%). Em 14 membros (71,5\%), a arcada posicionava-se proximalmente à $\mathrm{AF}$, em contato direto com o NIP. Em 2 membros (9,5\%), esta arcada tendinosa foi encontrada no mesmo nível da $\mathrm{AF}$, e em 4 membros (19\%) estava em uma posição distal sem contato direto com o NIP. Nossos achados se aproximam dos relatados por esses autores; identificamos a presença de uma arcada de composição fibrosa em 18 membros (60\%) e muscular em 9 (30\%); e em 3 (10\%) membros havia apenas a inserção radial, de maneira que não formava a arcada (-Fig. 4B). Em 17 dos 30 membros (57\%) identificamos a arcada do músculo ERCC posicionando-se proximalmente à AF, portanto em contato direto com o NIP, apoiando sobre ele, mas em apenas 5 (16,5\%) notamos uma arcada com espessura e estrutura macroscópica visível com consistência convincente para comprimir o NIP durante o movimento de pronação e supinação. Em 6 membros (20\%), identificamos a arcada posicionar-se distalmente à $\mathrm{AF}$, e em quatro $(13,5 \%)$ no mesmo nível da $\mathrm{AF}$, de forma que se apoiava sobre o $\mathrm{S}$ e não sobre o NIP (-Fig. 5A).

Sugerimos que a abordagem cirúrgica para descompressão do túnel radial pode ser feita com o antebraço em pronação, com uma incisão de $\sim 10 \mathrm{~cm}$ de comprimento, partindo do epicôndilo lateral acompanhando o eixo do rádio. Incisa-se a fáscia do antebraço e identifica-se o espaço entre o ERCC e o extensor dos dedos (ED). A dissecção é aprofundada neste espaço, identificando-se a AF e a arcada do músculo ERCC (-Tabelas 1 e 2 ).

Tabela 2 Sumário da literatura envolvendo a incidência da arcada do músculo extensor radial curto do carpo de acordo com os autores:

\begin{tabular}{|c|c|c|c|c|c|c|}
\hline Autores & $\begin{array}{l}\text { Arcada ERCC } \\
\text { fibrosa }\end{array}$ & $\begin{array}{l}\text { Arcada ERCC } \\
\text { muscular }\end{array}$ & $\begin{array}{l}\text { Arcada ERCC } \\
\text { ausente }\end{array}$ & $\begin{array}{l}\text { Tipo de } \\
\text { estudo }\end{array}$ & $\begin{array}{l}\text { número } \\
\text { de casos }\end{array}$ & ano \\
\hline Papadopoulos et al ${ }^{10}$ & $108(90 \%)$ & $12(10 \%)$ & - & anatômico & 120 & 1989 \\
\hline Konjengbam et al $^{15}$ & $36(78 \%)$ & $10(22 \%)$ & - & anatômico & 46 & 2004 \\
\hline Clavert $^{5}$ & $04(13 \%)$ & $26(87 \%)$ & - & anatômico & 30 & 2009 \\
\hline Nayak et al ${ }^{17}$ & $21(29,1 \%)$ & $4(13 \%)$ & $43(59,7 \%)$ & anatômico & 72 & 2010 \\
\hline Vergara-Amador et al $^{1}$ & $20(95,2 \%)$ & $01(4,8 \%)$ & - & anatômico & 21 & 2015 \\
\hline Caetano et al* & $18(60 \%)$ & $9(30 \%)$ & $3(10 \%)$ & anatômico & 30 & 2018 \\
\hline
\end{tabular}

Abreviação: ERCC, músculo extensor radial curto do carpo.

*Dados deste manuscrito. 
$\mathrm{O}$ NIP, proximalmente à $\mathrm{AF}$, pode ser identificado pela palpação contra a diáfise do rádio. A descompressão das duas arcadas deve ser feita.

\section{Conclusão}

A AF e a arcada formada pelas origens do músculo ERCC são estruturas anatômicas normais em cadáveres adultos. É importante ressaltar que, sob o ponto de vista clínico, essas estruturas têm potencial para causar a compressão do NIP.

\section{Conflito de Interesses}

Os autores declaram não haver conflito de interesses.

\section{Referências}

1 Vergara-Amador E, Ramírez A. Anatomic study of the extensor carpi radialis brevis in its relation with the motor branch of the radial nerve. Orthop Traumatol Surg Res 2015;101(08):909-912

2 Spinner M. The arcade of Frohse and its relationship to posterior interosseous nerve paralysis. JBone Joint Surg $\mathrm{Br}$ 1968;50(04): 809-812

3 Rinker B, Effron CR, Beasley RW. Proximal radial compression neuropathy. Ann Plast Surg 2004;52(02):174-180, discussion 181-183

4 Frohse F, Fränkel M. Die muskeln des menschlichen armes. In: Bardeleben $\mathrm{K}$, editor. Handbuch der anatomie des menschen. Jena: Gustav Fischer; 1908:164-165

5 Clavert P, Lutz JC, Adam P, Wolfram-Gabel R, Liverneaux P, Kahn JL. Frohse's arcade is not the exclusive compression site of the radial nerve in its tunnel. Orthop Traumatol Surg Res 2009;95(02): $114-118$
6 Ozturk A, Kutlu C, Taskara N, Kale AC, Bayraktar B, Cecen A. Anatomic and morphometric study of the arcade of Frohse in cadavers. Surg Radiol Anat 2005;27(03):171-175

7 Kopell HP, Thompson WAL. Peripheral entrapment neuropathies. Baltimore: William and Wilkins; 1963

8 Werner CO. Lateral elbow pain and posterior interosseous nerve entrapment. Acta Orthop Scand Suppl 1979;174:1-62

9 Lister GD, Belsole RB, Kleinert HE. The radial tunnel syndrome. J Hand Surg Am 1979;4(01):52-59

10 Papadopoulos N, Paraschos A, Pelekis P. Anatomical observations on the arcade of Frohse and other structures related to the deep radial nerve. Anatomical interpretation of deep radial nerve entrapment neuropathy. Folia Morphol (Praha) 1989;37(03):319-327

11 Prasartritha T, Liupolvanish P, Rojanakit A. A study of the posterior interosseous nerve (PIN) and the radial tunnel in 30 Thai cadavers. J Hand Surg Am 1993;18(01):107-112

12 Riffaud L, Morandi X, Godey B, et al. Anatomic bases for the compression and neurolysis of the deep branch of the radial nerve in the radial tunnel. Surg Radiol Anat 1999;21(04):229-233

13 Ozkan M, Bacakoğlu AK, Gül O, Ekin A, Mağden O. Anatomic study of posterior interosseous nerve in the arcade of Frohse. J Shoulder Elbow Surg 1999;8(06):617-620

14 Ebraheim NA, Jin F, Pulisetti D, Yeasting RA. Quantitative anatomical study of the posterior interosseous nerve. Am J Orthop 2000;29(09):702-704

15 Konjengbam M, Elangbam J. Radial nerve in the radial tunnel: anatomic sites of entrapment neuropathy. Clin Anat 2004;17(01): 21-25

16 Meng S, Tinhofer I, Weninger WJ, Grisold W. Ultrasound and anatomical correlation of the radial nerve at the arcade of Frohse. Muscle Nerve 2015;51(06):853-858

17 Nayak SR, Ramanathan L, Krishnamurthy A, et al. Extensor carpi radialis brevis origin, nerve supply and its role in lateral epicondylitis. Surg Radiol Anat 2010;32(03):207-211 